\title{
Driving Cycle Adaption and Design Based on Mean Tractive Force ${ }^{\star}$
}

\author{
Peter Nyberg* Erik Frisk* Lars Nielsen* \\ * Department of Electrical Engineering Linköping University, \\ SE-58183 Linköping, Sweden (e-mail: \{petny, frisk, lars\}@isy.liu.se).
}

\begin{abstract}
Driving cycles are used for certification, for comparison of vehicles, and to an increasing extent as an engineering tool in vehicle design. A situation with only a few fixed driving cycles to use would then lead to the risk that a test or design would be tailored to details in the driving cycle instead of being representative. Due to this, and due to the increased use in the development process, there is now a strong need for methods to achieve representative driving cycles that in a wide sense are similar but not the same. To approach this problem area, we define equivalence between driving cycles based on mean tractive force, and develop algorithms and methods for equivalence-modification and equivalence-transformation of driving cycles. There are a number of applications for these methods but one example that is demonstrated is to transform the well-known FTP75 into an equivalent NEDC, and the other way around, to transform the NEDC into an equivalent FTP75.
\end{abstract}

Keywords: Drive cycle, vehicle operation, driver behavior, vehicle propulsion, automotive emissions, test procedures, specific energy, equivalence measures.

\section{INTRODUCTION}

A driving cycle is a representation of how vehicles are driven and is usually represented by a set of data points of vehicle speed versus time. It is used for certification, for comparison of vehicles, or as an engineering tool in vehicle design (André, 1996), (Lin and Niemeier, 2002), or (Stockar et al., 2010). Regardless of application, the general idea behind a driving cycle is that it should be representative for driving behavior in the region it is used, and perhaps also that it should capture different driver behavior and different traffic situations. A representative driving cycle usually means that some statistical criteria of interest is sufficiently close to data from real-world driving.

There are many examples of driving cycles, and as an example a common one, is the New European Driving Cycle (NEDC), is seen in Figure 1. This particular driving cycle has low levels of acceleration compared to several examples of real-world driving, and thus, the NEDC is not representative of real-world driving conditions (Zaccardi and Le Berr, 2012). The NEDC also underestimate the $\mathrm{CO}_{2}$ emissions compared to real-world driving (Fontaras and Dilara, 2012). Further, design parameters that are optimized for a certain driving cycle are not necessarily optimal for another driving cycle (Schwarzer and Ghorbani, 2013), and a reason for this is that vehicle manufacturers need only to focus on limited operating regions of the engine (Kàgeson, 1998). If another driving cycle excites the operating regions differently, different exhaust gas emissions and fuel consumption characteristics are obtained, and thus if the driving cycle is not representative, the optimization on a single driving cycle, will be a suboptimal solution for real-world driving (Schwarzer and Ghorbani, 2013; Kågeson, 1998). With this background, and the increased importance of using driving cycles in

\footnotetext{
* This work was partially supported by the Swedish Research
} Council within the Linnaeus Center CADICS.

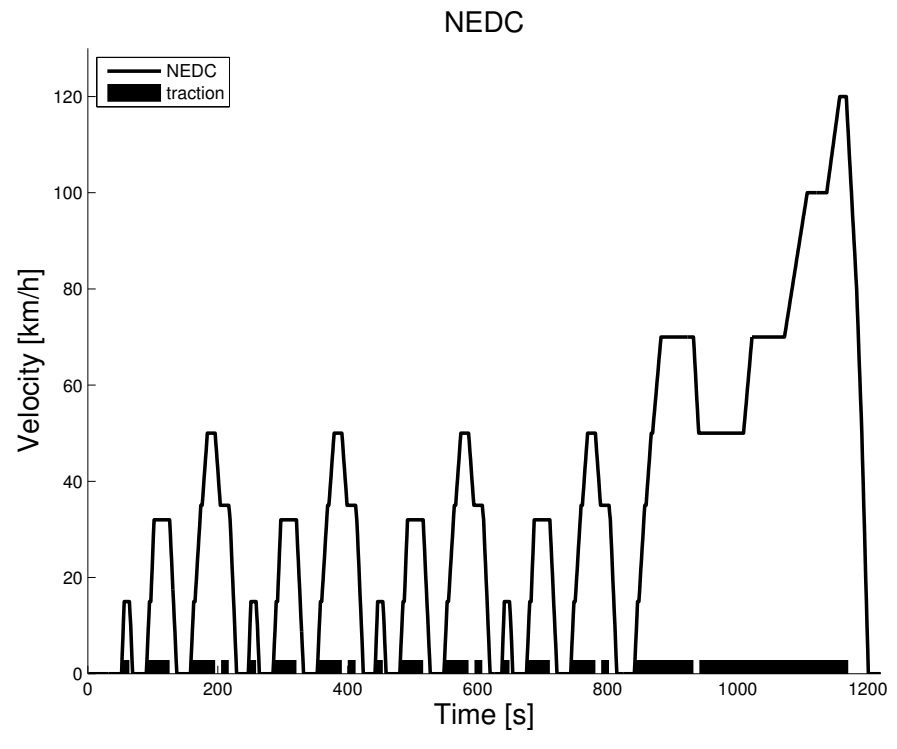

Fig. 1. The NEDC with marked traction regions which indicates the instants where the powertrain needs to deliver positive power to the wheels so that the vehicle is able to track the driving cycle.

the vehicle product development process, it is natural that there are new proposals for finding representative driving cycles, see for example (Lee and Filipi, 2011), (Lin and Niemeier, 2002), (Schwarzer and Ghorbani, 2013), or (Kamble et al., 2009).

To avoid the single cycle problem, it would be highly beneficial to have mechanisms to generate similar driving cycles that are not the same. The approach taken here is to define the concept of equivalent driving cycles, and this is done by using integral measures on the driving cycles, with mean tractive force (MTF) (Guzzella and Sciarretta, 2007) as the main example in this paper. 
The main objective is to find algorithms that can modify a given driving cycle into a different driving cycle that is equivalent in the measure, or to modify a given driving cycle into another one with a specified equivalence measure. Formulating and approaching these problems are the main topic of this paper.

\section{DRIVING CYCLE EQUIVALENCE}

A driving cycle excites and tests vehicles, and a vehicle's particular parameters determine the impact the driving cycle has on the powertrain. To compare the performance obtained in two different driving cycles is difficult since the cycles have different characteristics. A naive way would be to scale the driving cycles so they have the same mean vehicle speed. However, this does not make the cycles equivalent, since acceleration characteristics may still be radically different. Thus, a more insightful characterization of driving cycles is needed.

\subsection{Mean Tractive Force Equivalence}

The required torque at the wheels, from acceleration, is higher in the FTP75 compared to the NEDC since the FTP75 has more aggressive acceleration sequences. The EUDC (the highway part of the NEDC), has higher mean velocity than the FTP75 which yields that the rolling resistance, which is dependent on the rolling coefficient of the tires of the vehicle, has a higher impact on the vehicle power. Hence, the torque and power are dependent on the driving cycle, the vehicle mass and other parameters of the vehicle.

Using a measure on a driving cycle it is possible to define equivalence among driving cycles and also to make comparison between cycles possible. A measure can be defined on the whole time interval $\tau=\left[0, t_{\text {final }}\right]$, but the characterization used here is based on the mean tractive force (MTF) (Guzzella and Sciarretta, 2007). The integration intervals for MTF are determined by the vehicle traction regions where the powertrain of the vehicle provides positive power $(F(t)>0)$, i.e. $\bar{F}_{\text {trac }}$ is defined on a subset $\tau_{\text {trac }}=\{t \in \tau: F(t)>0\}$, and the measure is

$$
\bar{F}_{\text {trac }}=\frac{1}{x_{\text {tot }}} \int_{t \in \tau_{\text {trac }}} F(t) \cdot v(t) d t,
$$

where $x_{\text {tot }}$ is the distance traveled in the driving cycle, $F(t)$ is the sum of the forces at the wheels, and $v(t)$ is the speed of the vehicle. The measure (1) can be used for a preliminary estimate of the fuel consumed by the propulsion system (Guzzella and Sciarretta, 2007).

For flat roads the forces at the wheels origins from aerodynamic drag resistance force $\left(F_{\text {air }}\right)$, rolling resistance force $\left(F_{\text {roll }}\right)$, and also forces due to acceleration/deceleration of the vehicle $\left(F_{\mathrm{m}}\right)$. These forces are here modeled as

$$
\begin{aligned}
F(t) & =F_{\text {air }}+F_{\text {roll }}+F_{\mathrm{m}} \\
F_{\text {air }} & =\frac{1}{2} \rho_{a} c_{d} A_{f} v^{2}(t) \\
F_{\text {roll }} & =m c_{r} g \\
F_{\mathrm{m}} & =m a(t),
\end{aligned}
$$

where $\rho_{a}$ is the air density, $c_{d}$ is the drag coefficient, $A_{f}$ is the frontal area. The rolling resistance is modeled as proportional to the vehicle weight. The weight is the product of vehicle mass $(m)$, and the gravitational constant $g$. The proportional constant is denoted $c_{r}$. Finally, the force from acceleration is the mass times the acceleration $a(t)$.
The MTF quantity $\bar{F}_{\text {trac }}$ in (1), can be partitioned into three different parts originating from aerodynamic drag resistance, rolling resistance, and acceleration resistance,

$$
\begin{aligned}
\bar{F}_{\text {trac }} & =\bar{F}_{\text {air }}+\bar{F}_{\text {roll }}+\bar{F}_{\mathrm{m}} \\
\bar{F}_{\text {air }} & =\frac{1}{x_{\text {tot }}} \int_{t \in \tau_{\text {trac }}} \frac{1}{2} \rho_{a} c_{d} A_{f} v^{2}(t) \cdot v(t) d t=\kappa \alpha \\
\bar{F}_{\text {roll }} & =\frac{1}{x_{\text {tot }}} \int_{t \in \tau_{\text {trac }}} m g c_{r} \cdot v(t) d t=m g c_{r} \beta \\
\bar{F}_{\mathrm{m}} & =\frac{1}{x_{\text {tot }}} \int_{t \in \tau_{\text {trac }}} m a(t) \cdot v(t) d t=m \gamma,
\end{aligned}
$$

where $\kappa=\frac{1}{2} \rho_{a} c_{d} A_{f}$. The force components in (4-6) have been split into two parts where the first part consists of constants and vehicle parameters. The other part is driving cycle specific, but is however influenced by the vehicle parameters indirectly due to their influence on the coasting velocity in (10), which affects the traction regions, $\tau_{\text {trac }}$. The parameters related to the driving cycle are

$$
\begin{aligned}
\alpha & =\frac{1}{x_{\mathrm{tot}}} \int_{t \in \tau_{\mathrm{trac}}} v^{2}(t) \cdot v(t) d t \\
\beta & =\frac{1}{x_{\mathrm{tot}}} \int_{t \in \tau_{\mathrm{trac}}} 1 \cdot v(t) d t=\frac{x_{\mathrm{trac}}}{x_{\mathrm{tot}}} \\
\gamma & =\frac{1}{x_{\mathrm{tot}}} \int_{t \in \tau_{\mathrm{trac}}} a(t) \cdot v(t) d t .
\end{aligned}
$$

Based on the introduced MTF in $(4-6)$ we are ready to define an equivalence measure for driving cycles

Definition 1. For a given vehicle, two driving cycles, $v_{1}(t)$ and $v_{2}(t)$, are said to be equivalent, denoted $v_{1}(t) \sim v_{2}(t)$, if the following are fulfilled

$$
\begin{aligned}
& \alpha\left(v_{1}(t)\right)=\alpha\left(v_{2}(t)\right) \\
& \beta\left(v_{1}(t)\right)=\beta\left(v_{2}(t)\right) \\
& \gamma\left(v_{1}(t)\right)=\gamma\left(v_{2}(t)\right) .
\end{aligned}
$$

\subsection{Determining Traction Regions}

A vehicle is in traction if the powertrain need to provide positive power $(F(t)>0$ in $(2))$. The other two modes are coasting $(F(t)=0)$ and braking $(F(t)<0)$. Another way to determine if the vehicle is in traction is to calculate the coasting velocity which is determined by (2). Using $\dot{v}(t)=a(t)$ and $F(t)=0$, the vehicle coasting speed $\left(v_{\text {coast }}\right)$ is determined by

$$
\begin{aligned}
0 & =F_{\text {air }}+F_{\text {roll }}+F_{\mathrm{m}} \Rightarrow \\
\dot{v}_{\text {coast }}(t) & =-\frac{1}{2 m} \rho_{a} c_{d} A_{f} v_{\text {coast }}^{2}(t)-c_{r} g \\
& =-k_{1}^{2} v_{\text {coast }}^{2}(t)-k_{2}^{2} \Rightarrow \\
v_{\text {coast }}(t) & =\frac{k_{2}}{k_{1}} \tan \left(\arctan \left(\frac{k_{1}}{k_{2}} v_{\text {coast }}\left(t_{0}\right)\right)-k_{1} k_{2} \cdot t\right) .
\end{aligned}
$$

For discrete driving cycles and given an initial speed $v\left(t_{0}=\right.$ $\left.t_{k-1}\right)$ in the driving cycle, the vehicle is in traction at time $t_{k}$ if $v\left(t_{k}\right)>v_{\text {coast }}\left(t_{k}\right)$, otherwise the vehicle is in coasting $\left(v\left(t_{k}\right)=v_{\text {coast }}\left(t_{k}\right)\right)$, or in braking $\left(v\left(t_{k}\right)<v_{\text {coast }}\left(t_{k}\right)\right)$. The traction regions in the NEDC for a certain vehicle is seen as the marked regions near the x-axis in Figure 1.

\subsection{Physical Interpretation of the MTF Components}

The parameters $\beta$ and $\gamma$ have physical meanings as follows. The parameter $\beta$ in (8), which is related to the MTF measure for the rolling resistance, is the quotient between 
the distance traveled during traction, $x_{\text {trac }}$, and the total driven distance $x_{\text {tot }}$.

The MTF component (6) can be written as

$$
\begin{aligned}
\bar{F}_{\mathrm{m}}=m \gamma & =\frac{1}{x_{\mathrm{tot}}} \int_{t \in \tau_{\mathrm{trac}}} m \dot{v}(t) \cdot v(t) d t \\
& =\frac{1}{x_{\mathrm{tot}}} \int_{t \in \tau_{\mathrm{trac}}} \frac{m}{2} \cdot \frac{d v^{2}(t)}{d t} d t \\
& =\frac{1}{x_{\mathrm{tot}}} \sum_{i}^{\# \operatorname{trac}}\left[\frac{m \cdot v^{2}(t)}{2}\right]_{t_{i, \mathrm{start}}}^{t_{i, \mathrm{end}}},
\end{aligned}
$$

where it is clearly seen that it is proportional to the sum of the difference in kinetic energy for all the traction intervals. The number of traction intervals is denoted \#trac and each traction interval has its start, $t_{i, \text { start }}$, and end point $t_{i, \text { end }}$. Given that $x_{\text {tot }}$ is the same for two driving cycles, they have the same $\bar{F}_{\mathrm{m}}$ if $\sum_{i}^{\# \operatorname{trac}}\left[\frac{m \cdot v^{2}(t)}{2}\right]_{t_{i, \text { start }}}^{t_{i, \text { end }}}$ are equal. If the start and end points of the traction regions are not changed and $x_{\text {tot }}$ is kept constant this measure will be the same before as after any changes in the driving cycle. This observation will be used in the algorithms further on.

\section{PROBLEM FORMULATION}

A key problem studied is how to transform a given driving cycle, into another driving cycle that is equivalent in the sense of Definition 1. The first basic problem is thus

Problem P0: Given a driving cycle $v(t)$, find $\tilde{v}(t) \neq v(t)$ so that $\tilde{v}(t) \sim v(t)$.

A second strongly related problem is to incorporate a specified driving cycle segment, $v_{\text {spec }}$ defined on $\tau_{\text {spec }} \subset \tau$, within the driving cycle, where $\tilde{v}(t)=v_{\text {spec }}(t), t \in \tau_{\text {spec }}$.

Problem P1: $\quad$ Given a driving cycle $v(t)$, find $\tilde{v}(t) \neq v(t)$ so that (i) $\tilde{v}(t) \sim v(t)$ and (ii) $\tilde{v}(t)=v_{\text {spec }}(t), t \in \tau_{\text {spec }}$.

Another problem is when a driving cycle is given, but it is desired to change the excitation of the vehicle properties, while maintaining as much of the vehicle speed characteristics of the original driving cycle as possible. This can be done by changing the MTF quantity to the new desired target values $\left(\alpha^{\prime}, \beta^{\prime}, \gamma^{\prime}\right)$. The problem formulation is

Problem P2: Given a driving cycle $v(t)$ and a target $\left(\alpha^{\prime}, \beta^{\prime}, \gamma^{\prime}\right)$. Find $\tilde{v}(t)$ so that $\left|\alpha(\tilde{v}(t))-\alpha^{\prime}\right| \leq \epsilon_{\alpha}, \mid \beta(\tilde{v}(t))-$ $\beta^{\prime}\left|\leq \epsilon_{\beta},\right| \gamma(\tilde{v}(t))-\gamma^{\prime} \mid \leq \epsilon_{\gamma}$.

The target MTF could be the values for another driving cycle $v_{2}(t),\left(\alpha\left(v_{2}(t)\right), \beta\left(v_{2}(t)\right), \gamma\left(v_{2}(t)\right)\right)$, and the resulting driving cycle, $\tilde{v}(t)$, should resemble real-world driving and not be unrealistic. The next section will describe algorithms for the above mentioned problems.

\section{ALGORITHM}

A trivial solution to alter the driving cycle while maintaining the same MTF components would be to shuffle around the micro-trips to create a new driving cycle. This cycle is obviously altered but it is basically the same cycle. Instead we seek a solution that alter the driving cycle by doing iterative changes and at the same time maintaining the MTF quantities (3-6). To simplify the derivation of the algorithms, the total distance traveled, $x_{\text {tot }}$, and the traction regions will not be changed.

\subsection{Core Component: Analytical Local Modifications}

Denote the original driving cycle as $v_{0}$ (where the time dependence is omitted) and a slightly altered driving cycle, $\tilde{v}$, with a speed difference vector $\delta v$ that contains $n$ nonzero elements, $\delta v_{i}=\delta v\left(t_{i}\right)=\delta_{i}, i=1,2, \ldots, n$. The altered driving cycle is thus $\tilde{v}=v_{0}+\delta v$. The algorithm will not change the start or end points of traction intervals. This results in that the MTF component (6) is kept constant according to (11). This yields that only the integrals in $(7$ - 8) need to be considered, and thus two driving cycles has the same MTF if

$$
\begin{aligned}
\int_{t \in \tau_{\text {trac }}} v_{0}^{3} d t & =\int_{t \in \tau_{\text {trac }}}\left(v_{0}+\delta v\right)^{3} d t \\
\int_{t \in \tau_{\text {trac }}} v_{0} d t & =\int_{t \in \tau_{\text {trac }}}\left(v_{0}+\delta v\right) d t .
\end{aligned}
$$

It is possible to alter two speed points $\left(v_{01}, v_{02}\right)$ and still maintain the MTF. However, the solution is then the trivial solution, the speed points change position $\left(\tilde{v}_{01}=\right.$ $v_{02}$ and $\left.\tilde{v}_{02}=v_{01}\right)$. Hence, switching or sorting speed points will not change the MTF as long the traction modes remain unchanged, and the start and end points in each traction region is not changed.

An issue is that the speed difference vector, $\delta v$, is not allowed to be chosen freely. The fact that the traction regions shall be intact implies that a change in the driving cycle is forced to not change the traction mode for any points. An illustration of the boundaries for any change in speed $v\left(t_{k}\right)$ is seen in Figure 2. The upper boundary origins from a limitation in the maximum acceleration, $a_{\max }$, at point $v\left(t_{k}\right)$ and also that the next speed point $v\left(t_{k+1}\right)$ does not lose traction. The lower boundary comes from that the current speed point will not lose traction and that the acceleration for the next speed point is kept within the maximum acceleration.

If three speed points are considered $(n=3)$, an extra degree of freedom is introduced. The variable that is free is $\delta_{2}$, and when it is set it determines $\delta_{1}$ and $\delta_{3}$. For discrete driving cycles, the solution of (12-13) are

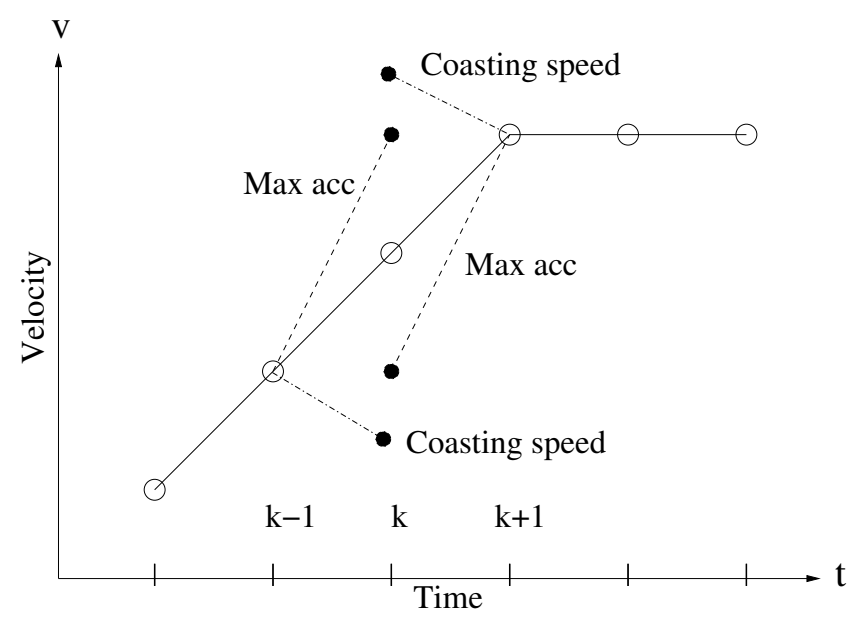

Fig. 2. Limitations of changes of $v\left(t_{k}\right)$ which origins from maintaining the traction regions and also considering that the maximum acceleration is not exceeded. 


$$
\begin{aligned}
\sum v_{0} & =\sum v_{0}+\delta v \Rightarrow \\
\delta_{3} & =-\left(\delta_{1}+\delta_{2}\right) \\
\sum v_{0}^{3} & =\sum\left(v_{0}+\delta v\right)^{3} \Rightarrow \\
0 & =\delta_{1}^{2} \cdot a\left(\delta_{2}\right)+\delta_{1} \cdot b\left(\delta_{2}\right)+c\left(\delta_{2}\right) \Rightarrow \\
\delta_{1} & =\frac{b}{2 a} \pm \sqrt{\frac{b^{2}}{4 a^{2}}-\frac{c}{a}},
\end{aligned}
$$

where $\delta_{2}$ is the free variable and its dependency on the factors a, b, and c is omitted in (15). Thus, by picking a suitable $\delta_{2}$ (considering Figure 2) and using (14-15), an altered driving cycle is achieved without changing the MTF measures. The reason for this is that the traction regions are intact, and (14) results in that the average speed is unchanged, hence the distance traveled during the traction regions is kept constant. Thus, $x_{\text {tot }}$ will not be changed and the measures (3-6) will be the same.

The core component is this analytical solution to make local modifications of a given driving cycle without changing the MTF. In the most straightforward way the three speed points are randomly selected. This can be iteratively done and the results would be a driving cycle that varies around the original driving cycle, and thus, a solution to problem $\mathrm{P} 0$. In the next section this core component will be used to make global modifications of the driving cycle.

\subsection{Algorithm 1: Global Modifications of the Driving Cycle}

Using the solution from the previous section iteratively produces an altered driving cycle that is of little use. It has the same MTF measures but due to the random selection of the speed points the resulting driving cycle is the original one with some fluctuations within it.

However, if the points that are changed in each iteration would be chosen more wisely, it is possible to alter the driving cycle in a more systematic way. For example, given a driving cycle, $v$, and a specified segment of a driving cycle, $v_{\text {spec }}$, with corresponding time points $\tau_{\text {spec }}$, Algorithm 1 will produce an equivalent driving cycle $\tilde{v}$ such that $\tilde{v}(t)=v_{\text {spec }}(t), t \in \tau_{\text {spec }}$, and hence, a solution for problem $\mathrm{P} 1$. The algorithm uses the analytical solution in (14-15) as a base to change the driving cycle iteratively until the specified driving cycle segment is achieved. The pseudo-code for Algorithm 1 is seen in Figure 3.

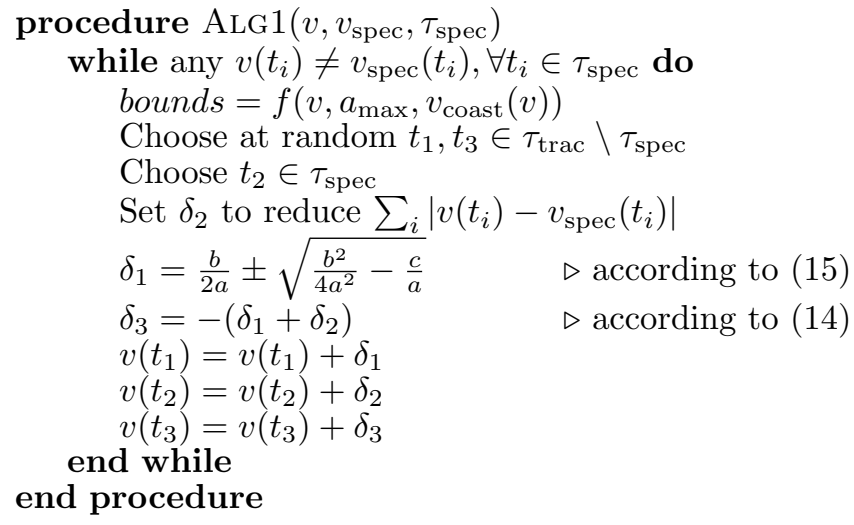

Fig. 3. Algorithm 1 change a given driving cycle, $v$, iteratively, so that a specified driving cycle segment is incorporated within $v$, while maintaining the MTF.

\subsection{Algorithm 2: Transforming to Target $\alpha, \beta$, and $\gamma$}

The core component and the previous algorithm consider only that the driving cycle should maintain the MTF. To change how a driving cycle excites the vehicle properties a novel algorithm is proposed that, iteratively changes one measure at the time until the MTF of the altered driving $\tilde{v}$ is sufficiently close to target values $\left(\alpha^{\prime}, \beta^{\prime}, \gamma^{\prime}\right)$.

Step 1: Transforming $\beta$ As previously discussed, the quantity $\beta$ in (8) is the ratio between traveled distance during traction regions and the total driven distance in the driving cycle. To change the driving cycle such that $\left|\beta(\tilde{v})-\beta^{\prime}\right| \leq \epsilon_{\beta}$ the speed points within the traction regions will be altered. This will change $x_{\text {trac }}$ in (8) until the difference between $\beta(\tilde{v})$ and $\beta^{\prime}$ is sufficiently small.

Step 2: Transforming $\gamma$ If the traction regions are intact and $x_{t o t}$ is held constant, it is only possible to alter $\gamma(\tilde{v})$ by changing the speed at the start and end of each traction interval. By iteratively changing the end points it is possible to achieve $\left|\gamma(\tilde{v})-\gamma^{\prime}\right| \leq \epsilon_{\gamma}$. Every change in the end points need to be compensated so that the average speed is kept constant and hence also $x_{t o t}$. If the traction region shall remain intact it may be necessary to scale certain traction intervals. Keeping $x_{t o t}$ constant is still vital for not changing $\beta(\tilde{v})$.

Step 3: Transforming $\alpha$ Finally, to modify the driving cycle to get $\left|\alpha(\tilde{v})-\alpha^{\prime}\right| \leq \epsilon_{\alpha}$ while maintaining both $\beta(\tilde{v})$ and $\gamma(\tilde{v})$, can be achieved by expanding or contracting of the speed points (keeping the average speed) in the driving cycle. If the average speed is kept constant and the traction regions are maintained it will result in that $x_{t o t}$ is the same and hence will not affect $\beta(\tilde{v})$. If addition the start and end points of each traction regions is kept constant this will neither affect $\gamma(\tilde{v})$.

Algorithm Summary The algorithm is summarized in Figure 4, and is a solution for problem P2. For illustration of the use of Algorithm 2, the NEDC, $v_{1}$, will be transformed to the target MTF of the FTP75, $v_{2}$. Hence, producing an altered version of the NEDC, denoted $\tilde{v}_{1}$ which has an MTF that is sufficiently close to the MTF of $v_{2}$. A comparison between the original NEDC, the output from the intermediate step 2 (after the second while-loop in Figure 4), and the output after the last step is seen in Figure 5. The dash-dotted line correspond to a driving cycle that has similar MTF components as the FTP75 for (8) and (9). The solid line correspond to a driving cycle that has similar MTF as the FTP75. As can be seen in the figure, the resulting driving cycle is unrealistic since it varies to much. To get a smooth driving cycle an MTF invariant low-pass filtering algorithm can be used and it will be explained in the next section.

\subsection{Algorithm for Reducing Fluctuations}

The algorithms that have been proposed have problems that the resulting driving cycle fluctuates to much and can be unrealistic. These problems origins from the random selection of the point, in the algorithms, that causes the acceleration from point to point to not be so smooth. To remedy this, we propose a filtering step that sorts the points in each traction interval so the change in acceleration from point to point is smoother. Such a smoothing of the driving cycle does not affect the MTF 


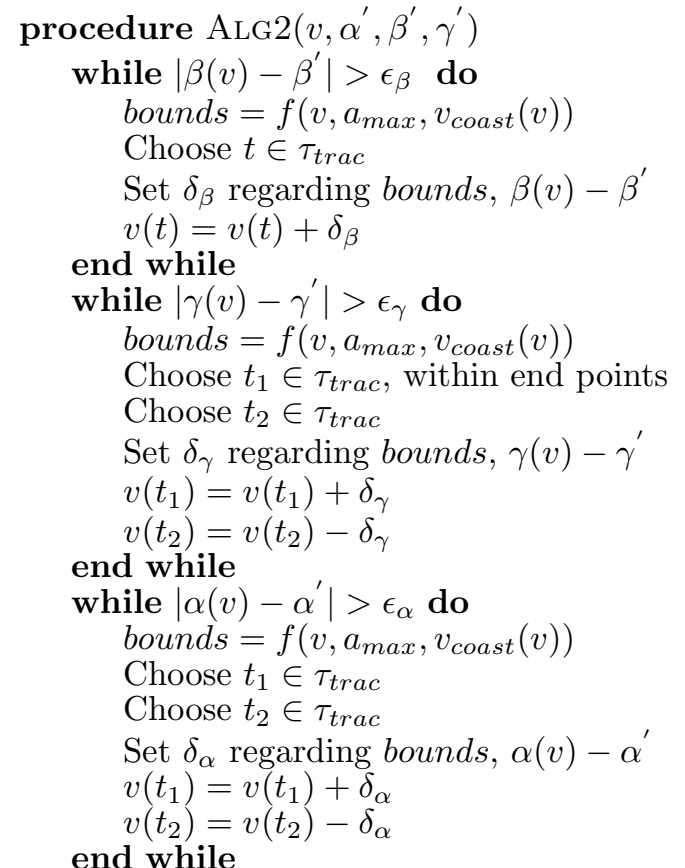

\section{end procedure}

Fig. 4. Algorithm 2 transform a driving cycle to pre-defined target measures $\left(\alpha^{\prime}, \beta^{\prime}, \gamma^{\prime}\right)$. The acceptable difference are determined by thresholds $\left(\epsilon_{\alpha}, \epsilon_{\beta}, \epsilon_{\gamma}\right)$.

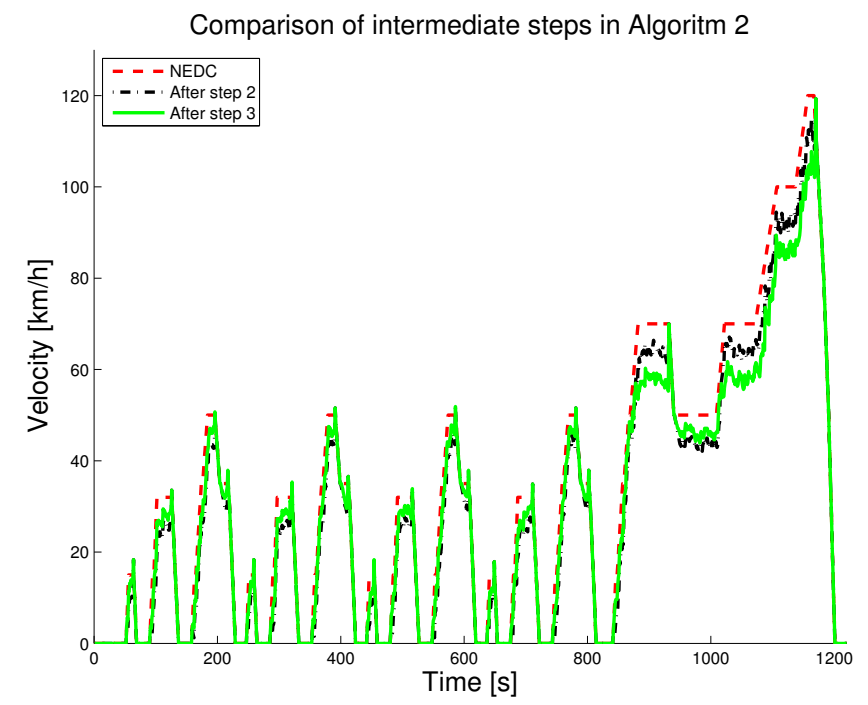

Fig. 5. Comparison between different stages in algorithm 2 where the NEDC is transformed so that it gets similar MTF as the FTP75. Intermediate step after transforming $\beta$ and $\gamma$ (dash-dotted), final step after also transforming $\alpha$ (solid) and the NEDC (dashed).

as long as the start and end points of each traction region are not altered and the traction region intervals are not changed.

The algorithm is applied on each traction interval and sorts and smooths the speed points depending on the speed of the start and end points, $v\left(t_{i, \text { start }}\right)$ and $v\left(t_{i, \text { end }}\right)$. For example, for the case $v\left(t_{i, \text { start }}\right) \leq v\left(t_{i, \text { end }}\right)$ and the interval $I_{i}=\left(t_{i, \text { start }}, t_{i, \text { end }}\right)$ the filtering becomes a sorting in ascending order if $v\left(t_{i, \text { start }}\right) \leq v(t) \leq v\left(t_{i, \text { end }}\right), \forall t \in I_{i}$. If there exists speed points and $t \in I_{i}$ such that

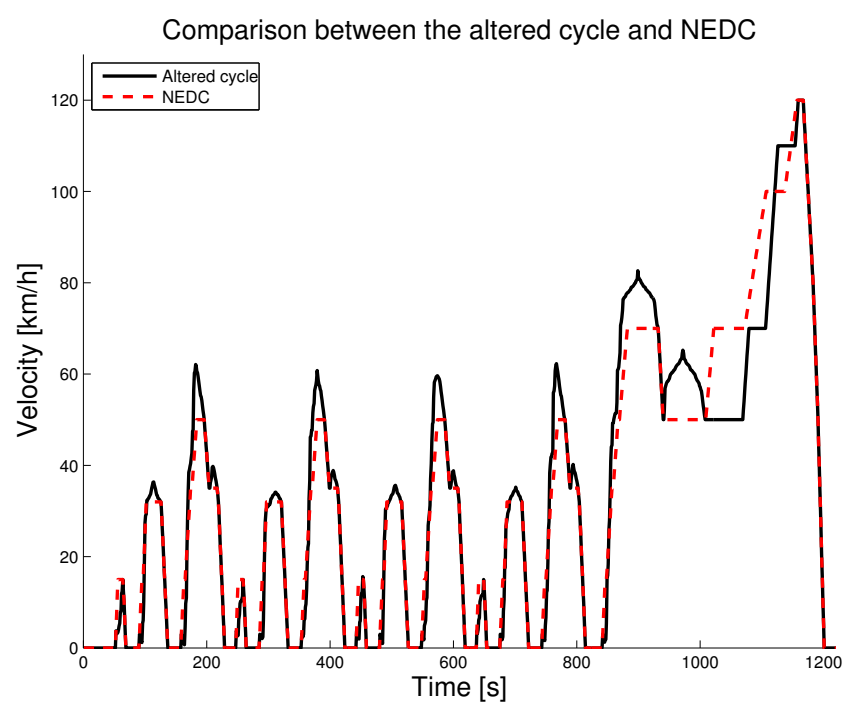

Fig. 6. Results from Algorithm 1 when a specified driving cycle segment is wanted within an existing driving cycle, while the MTF are kept constant.

$v\left(t_{i, \text { start }}\right)>v(t)$ or $v(t)>v\left(t_{i, \text { end }}\right)$ these points will be partitioned in such a way that the change in acceleration, from point to point, will be reduced in each traction interval. The outcome of the filtering step is seen in Figure 7 compared to the non-filtered (solid line) in Figure 5.

\section{CASE EXAMPLES}

Solving problems P0-P1, can be done using Algorithm 1. Assume, for the sake of illustration, that the highway part of the NEDC is wanted to be more aggressive to test some transient behavior, but the new test cycle should still be equivalent to NEDC. In the example, $\tau_{\text {spec }}=[1009,1180]$, and the new speed profile in that segment, $v_{\text {spec }}$, with more aggressive acceleration is seen in Figure 6 as the solid curve. In addition, the rest of the cycle, defined on $[0,1008]$, needs to be modified so that the complete driving cycle, defined on $[0,1180]$, still affects the vehicle parameters in a similar way. By giving $v_{\text {spec }}$ to Algorithm 1 the output will be a driving cycle that is equivalent to the NEDC, and that has the desired driving cycle segment, $v_{\text {spec }}$, within it during $\tau_{\text {spec }}=[1009,1180]$. The result of this, including a filtering step for reducing fluctuations, is seen in Figure 6.

Given a driving cycle, $v$, and specified equivalence measures $\left(\alpha^{\prime}, \beta^{\prime}, \gamma^{\prime}\right)$, Algorithm 2 transforms the driving cycle until the $\alpha(v), \beta(v)$, and $\gamma(v)$ are sufficiently close to target measures, and thus a solution for problem $\mathrm{P} 2$. For illustration of the use of Algorithm 2, the NEDC, $v_{1}(t)$, is transformed to the target measures of the FTP75, $v_{2}(t)$. The filtered results from Algorithm 2 is seen in Figure 7 . The values for the FTP75 for a certain vehicle are $\left(\alpha\left(v_{2}(t)\right), \beta\left(v_{2}(t)\right), \gamma\left(v_{2}(t)\right)\right)=$ $(231,0.83,0.14)$ and corresponding values for the NEDC are $\left(\alpha\left(v_{1}(t)\right), \beta\left(v_{1}(t)\right), \gamma\left(v_{1}(t)\right)\right)=(324,0.85,0.11)$. Due to $\beta\left(v_{1}(t)\right)>\beta\left(v_{2}(t)\right)$ the speed points during traction intervals have to be lowered, and this can also be seen in Figure 7 where the average speed of the equivalent driving cycle is lower than the original NEDC. When the first step in Algorithm 2 has been performed, the MTF measures have been changed. Denote $\gamma_{\text {step } 1}$ to be the $\gamma$ value after step 1 . Due to $\gamma_{\text {step } 1}<\gamma\left(v_{2}(t)\right)$ the end points of each traction region need to be raised. Finally due to 


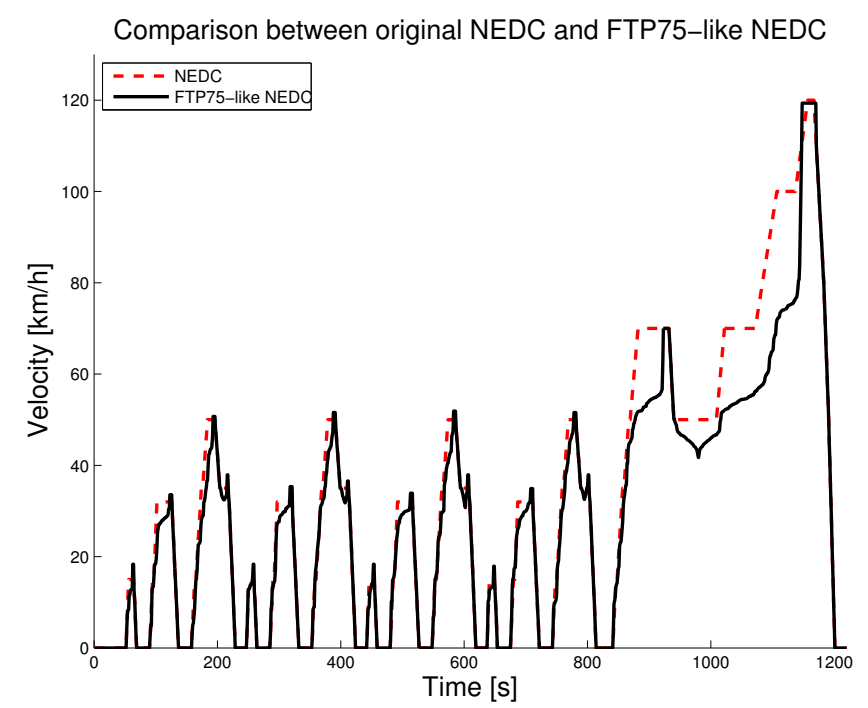

Fig. 7. Comparison between NEDC (dashed) and an altered driving cycle (solid) which has MTF values close, in the sense of $\left(\epsilon_{\alpha}, \epsilon_{\beta}, \epsilon_{\gamma}\right)$, to the FTP75.

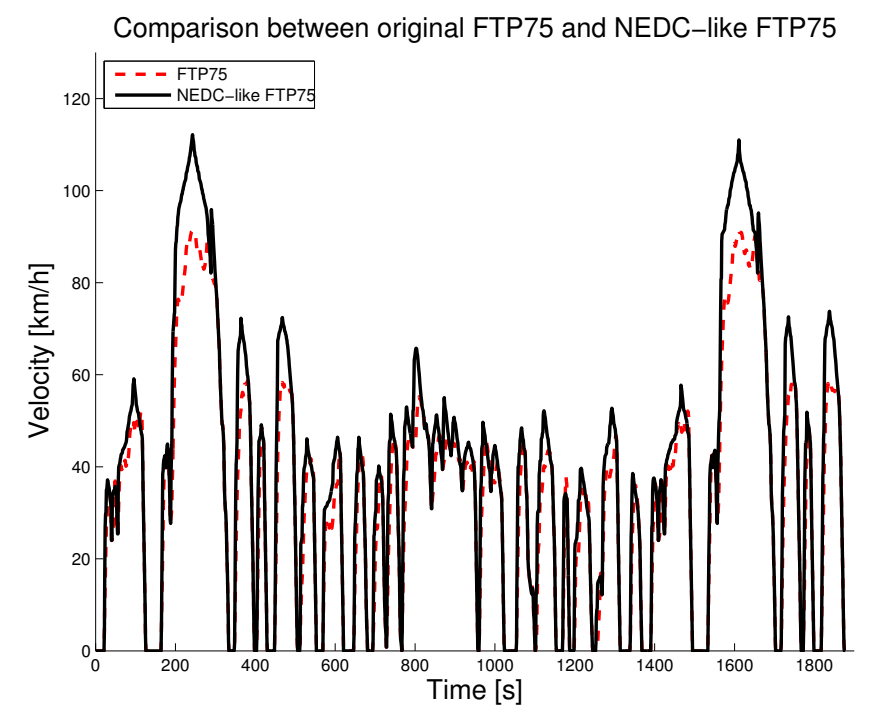

Fig. 8. Comparison between FTP75 (dashed) and an altered driving cycle (solid) which has MTF values close, in the sense of $\left(\epsilon_{\alpha}, \epsilon_{\beta}, \epsilon_{\gamma}\right)$, to the NEDC.

$\alpha_{\text {step } 2}>\alpha\left(v_{2}(t)\right)$, the speed during traction has to be contracted to fully be transformed to a driving cycle that has an MTF sufficiently close to the FTP75.

The other way around (from FTP75 to NEDC) is seen in Figure 8. Notice that due to $\beta\left(v_{1}(t)\right)>\beta\left(v_{2}(t)\right)$ this will result in that the average speed in the transformed FTP75 driving cycle will be raised even if the average speed of the NEDC is lower than the FTP75 from start. The reason for this is that (8) is proportional to the ratio between distance traveled in the traction regions and the total distance traveled. It is a fairer measure of the tractive power at the wheels than the average speed, which does not consider the coasting or braking regions.

\section{CONCLUSIONS}

Driving cycles are used for certification, for comparison of vehicles, and as an engineering tool in vehicle design. Due to the increased use and importance of driving cycles, especially in the vehicle development process, there is a strong need for methods to find or design representative driving cycles, but also to work with them and to modify them in the engineering process. A key step taken here was to define the concept of equivalent driving cycles based on mean tractive force. Using this measure two main problems were defined, namely how to modify a driving cycle into a different one but with the same equivalence measure, and how to modify a driving cycle so that its measure comes sufficiently close to a predefined equivalence measure. Algorithms solving these problems were presented, a core component where used for local modification and using this repeatedly achieved global modifications of the driving cycle. This fits well with the engineering situation when there is a representative driving cycle at hand, and the wish is to modify it slightly without loosing its characteristics. With our approach the level of modification can be controlled, and, furthermore, the modifications can be directed to parts of the driving cycle of specific interest. One such example is to request for a little different behavior in a certain transient, but where one would still like to have an MTF-equivalent driving cycle to get comparable results. Even though the algorithms are not yet optimized they show the feasibility of the methods and also gives interesting results. Two examples that were demonstrated was to transform the well-known FTP75 into an equivalent NEDC, and the other way around, to transform the NEDC into an equivalent FTP75. We foresee further development and more applications for the ideas and methods presented.

\section{REFERENCES}

André, M. (1996). Driving cycles development: Characterization of the methods. In SAE Technical Paper 961112.

Fontaras, G. and Dilara, P. (2012). The evolution of European passenger car characteristics 2000-2010 and its effects on real-world $\mathrm{CO}_{2}$ emissions and $\mathrm{CO}_{2}$ reduction policy. Energy Policy, 49(0), 719 - 730.

Guzzella, L. and Sciarretta, A. (2007). Vehicle Propulsion System: Introduction to Modeling and Optimization. Springer.

Kamble, S., Mathew, T., and Sharma, G. (2009). Development of real-world driving cycle: case study of Pune, India. Trans. Research Part D, 14(2), 132-140.

Kågeson, P. (1998). Cycle-beating and the EU test cycle for cars. European Fed. for Trans. and Envir. T\&E, $98(3)$.

Lee, T.K. and Filipi, Z. (2011). Synthesis of real-world driving cycles using stochastic process and statistical methodology. Int. J. Vehicle Design, 57(1), 17-36.

Lin, J. and Niemeier, D. (2002). An exploratory analysis comparing a stochastic driving cycle to California's regulatory cycle. Atmospheric Envir., 36, 5759-5770.

Schwarzer, V. and Ghorbani, R. (2013). Drive cycle generation for design optimization of electric vehicles. IEEE Transactions on Vehicular Technology, 62(1), 89 $-97$.

Stockar, S., Tulpule, P., Marano, V., and Rizzoni, G. (2010). Energy, economical and environmental analysis of plug-in hybrids electric vehicles based on common driving cycles. SAE Int. Journal of Engines, 2(2), 467476.

Zaccardi, J.M. and Le Berr, F. (2012). Analysis and choice of representative drive cycles for light duty vehicles case study for electric vehicles. Proc. of the Inst. of Mech. Engineers, Part D: J. of Automobile Engineering. 